ESAIM: PROCEEDINGS AND SURVEYS, September 2014, Vol. 45, p. 318-327

J.-S. Dhersin, Editor

\title{
BOREL-LAPLACE SUMMATION METHOD USED AS TIME INTEGRATION SCHEME
}

\author{
Ahmad Deeb $^{1}$, Aziz Hamdouni ${ }^{1}$, Erwan Liberge $^{1}$ And Dina Razafindralandy ${ }^{1}$
}

\begin{abstract}
A time integration method for the resolution of ordinary and partial differential equations is proposed. The method consists in computing a formal solution as a (eventually divergent) time series. Next, the Borel resummation method is applied to deduce an (sectorial) analytical solution. The speed and spectral properties of the scheme are analyzed through some examples. Applications to fluid mechanics are presented.

Résumé. On propose une méthode numérique d'intégration temporelle d'équations différentielles ou aux dérivées partielles. Cette méthode consiste d'abord à calculer une solution sous forme de série formelle, dont le rayon de convergence peut être nul. Ensuite, la méthode de resommation de BorelLaplace est utilisée pour déduire une solution analytique (dans un secteur) de l'équation. La rapidité et les propriétés géométriques du schéma sont analysées à travers quelques exemples. Des applications en mécanique des fluides sont présentées.
\end{abstract}

\section{INTRODUCTION}

Perturbation theory [5] is a widely used method to approximate solutions of physical problems. In particular, it has been successfully used as a numerical tool in engineering science for the detection of bifurcations, the regularization of constitutive laws, fracture problems, .. [ $[2,3,10,14]$. Recently, we proposed to develop a time integration scheme, based on the perturbation theory, for the numerical resolution of differential equations in mechanics, especially fluid mechanics [24]. To this aim, a formal solution is sought as a time series.

Care has however to be taken when manipulating time series since they are frequently divergent. Indeed, divergent series arise naturally in many areas of science, ranging from astronomy [19,23], through quantum physics $[16,26]$, to engineering science $[12,20]$. For example, the formal time-series solution of the heat equation or that of the Navier-Stokes equations are generally divergent. Fortunately, even diverging, the asymptotic series contains enough information on the actual solution. One way to compute effectively this actual solution from the divergent series is the Borel-Laplace resummation method $[6,7]$.

In [24], the Borel-Laplace resummation method has been transformed into a numerical algorithm and associated with the numerical asymptotic method [13] to give rise to an efficient time integration scheme. This scheme has the property to be explicit, in the sense that, at most, only one matrix inversion is needed. Moreover, the operator is automatically linearized. In the present paper, the speed of this scheme and its "spectral" qualities are investigated.

In Section 1, the Borel-Laplace theory and the numerical scheme are recalled. In Section 2, the scheme is analyzed from a qualitative and qualitative (geometric) point of view. Finally, SeCtion 3 is devoted to applications to fluid mechanics problems.

\footnotetext{
${ }^{1}$ Laboratoire des Sciences de l'Ingénieur pour l'Environnement (LaSIE), Université de La Rochelle. France.
}

(C) EDP Sciences, SMAI 2014 


\section{NumERICAL SCHEME}

Consider an ordinary differential or a partial derivative equation

$$
\frac{\partial u}{\partial t}=A(u), \quad u(0)=u_{0}
$$

having as formal solution a time-series

$$
\hat{u}(t)=\sum_{n=0}^{\infty} u_{n} t^{n} .
$$

As mentioned, the first step of the scheme is to compute numerically the series $\hat{u}(t)$. This step is, in many applications, straightforward since it simply consists in injecting (2) in equation (1). In many cases, for instance when the operator $A$ is a (differential) polynomial in $u$, it results in a recurrence relation of the form:

$$
u_{n+1}=A_{n+1}\left(u_{0}, \cdots, u_{n}\right), \quad n \in \mathbb{N}
$$

where $A_{n+1}$ is another (differential) polynomial operator in $u$.

Series (2) is either convergent or divergent. When (2) is divergent, the Borel-Laplace resummation procedure has to be applied. When (2) is convergent, it already provides an analytical solution inside the (possibly numerically very small) convergence disk. But even in this case, the resummation procedure will be applied since it will act as a prolongation method outside the convergence disk.

\subsection{The Borel-Laplace resummation method}

When (2) is divergent and $A$ is meromorphic, there exists a solution $\mathrm{S} \hat{u}(t)$ of equation (1), holomorphic in a sectorial neighborhood of the origin, and owning the series $\hat{u}(t)$ as asymptotics in Gevrey sense $[5,22]$. Assume, as it is usually the case for almost all problems met in physics, that $\hat{u}(t)$ is a Gevrey series, that is $\left|u_{n}\right|<C A^{n} t^{n} n$ ! for some constants $C$ and $A$. Then $\mathrm{S} \hat{u}(t)$ may be recovered by applying the Borel-Laplace method which can be described in three stages as follows:

- First, the Borel transform

$$
\mathrm{B} \hat{u}(\xi)=\sum_{n \geq 0} \frac{u_{n+1}}{n !} \xi^{n}
$$

is computed. This transformed series is analytical at the origin.

- Next, $\mathrm{B} \hat{u}(\xi)$ is analytically prolongated into a function $P(\xi)$ within a sectorial domain containing an infinite semi-line $d$ from the origin.

- Lastly, $\mathrm{S} \hat{u}(t)$ is obtained by computing the Laplace transform of $P$ along the semi-line $d$ :

$$
\mathrm{S} \hat{u}(t)=u_{0}+\int_{d} P(\xi) \mathrm{e}^{-\xi / t} d \xi
$$

The Laplace transform is the formal inverse of the Borel transform. These three stages are summarized in TABLE 1.

As an example, consider the Euler equation

$$
t^{2} \frac{\mathrm{d} u}{\mathrm{~d} t}+u=t
$$

The formal series solution is

$$
\hat{u}(t)=\sum_{k \geq 0}(-1)^{n} n ! t^{n+1}
$$




$$
\begin{aligned}
& \hat{u}(t)=\sum_{n \geq 0} u_{n} t^{n} \quad \stackrel{\text { asymptotics }}{\simeq} \mathrm{S} \hat{u}(t)=u_{0}+\int_{d} P(\xi) \mathrm{e}^{-\xi / t} d \xi \\
& \text { Borel } \downarrow \text { Laplace } \\
& \mathrm{B} \hat{u}(\xi)=\sum_{n \geq 0} \frac{u_{n+1}}{n !} \xi^{n} \stackrel{\text { Prolongation }}{\longrightarrow} P(\xi)
\end{aligned}
$$

TABLE 1. The 3-stage Borel-Laplace resummation method

This series is a divergent but a Gevrey series. Its Borel transform prolongates naturally into the analytical function $P(\xi)=(1+\xi)^{-1}$. Hence, the Borel sum $\mathrm{S} \hat{u}(t)$ along the positive semi-line reads:

$$
\mathrm{S} \hat{u}(t)=\int_{0}^{+\infty} \frac{1}{1+\xi} \mathrm{e}^{-\xi / t} \mathrm{~d} \xi
$$

This function is the solution of the Euler equation (6), holomorphic in the complex right half-plane.

The above presentation of the Borel resummation method is very minimalistic. More complete information on the theory can be found, for example, in $[11,17,21,22]$.

\subsection{Borel-Padé-Laplace algorithm}

Numerically, the terms of the series $\hat{u}(t)$ are computed up to an arbitrary, but finite, order $N$. The Borel transform $\mathrm{B} \hat{u}(\xi)$ is then known at order $N-1$. Its prolongation can be materialized with various methods [27]. The one chosen here is the Padé extrapolation $[8,9]$. This means that the truncated Borel transform $\mathrm{B} \hat{u}^{N}(\xi)$ is substituted with a rational function $P^{N}(\xi)$ having $\mathrm{B} \hat{u}^{N}(\xi)$ as Taylor asymptotics at the origin. Finally, the Laplace transform is performed with a Gauss-Laguerre quadrature. The resulting algorithm, called Borel-PadéLaplace algorithm, is summarized in TABLE 2 . In this table, the $\xi_{i}$ are the roots of the $N_{G}$-th Gauss-Laguerre polynomials, corresponding to the weights $w_{i}$.

$$
\begin{aligned}
& \hat{u}^{N}(t)=\sum_{n=0}^{N} u_{n} t^{n} \\
& \mathrm{~S} \hat{u}^{N}(t)=u_{0}+\sum_{i=1}^{N_{G}} \tilde{P}\left(\xi_{i}\right) w_{i} \\
& \text { Borel } \downarrow \\
& \mathcal{B} \hat{u}^{N}(\xi)=\sum_{n=0}^{N-1} \frac{u_{n+1}}{n !} \xi^{n} \longrightarrow \text { Padé } \quad P^{N}(\xi)=\frac{A_{0}+A_{1} \xi+\cdots+A_{N_{1}} \xi^{N_{1}}}{1+B_{1} \xi+\cdots+B_{N_{2}} \xi^{N_{2}}}
\end{aligned}
$$

TABLE 2. Borel-Padé-Laplace algorithm

As an illustration, the algorithm is applied to the resolution of Euler equation (6). The truncation order $N$ is set to 10 and $N_{G}=6$. As can be observed in Figure 1 (excerpt from [24]), the Borel-Padé-Laplace algorithm gives a much better approximation of the analytical solution (8) compared to the asymptotic series. In fact, for equation $(6), P^{N}(\xi)=P(\xi)$ as soon as $N \geq 2$. The only source of error is then the Gaussian quadrature. 


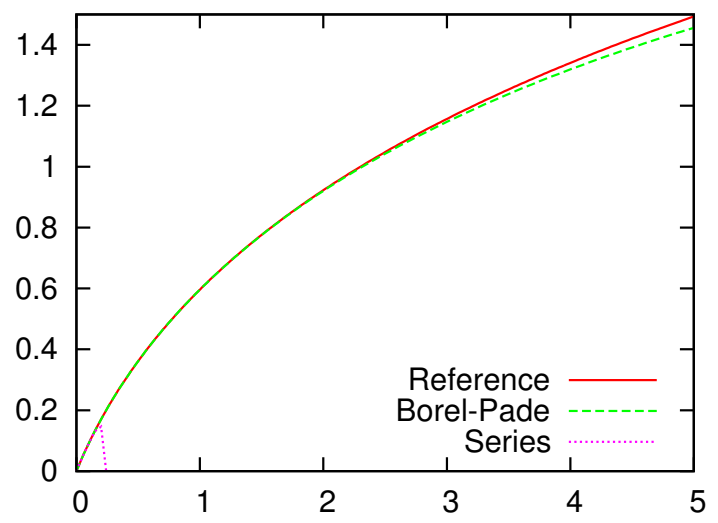

Figure 1. Time evolution of the approximate solutions of Euler equation

In the previous example, the original series is divergent. It has been shown in [24] on other equations that if the original series has a non vanishing convergence radius, the Borel-Padé-Laplace algorithm can be seen as a prolongation method, which provides an approximate solution outside the convergence disc. As a consequence, much less time iterations are needed.

In the next section, we will focus not only on the speed of the algorithm but also on qualitative aspects.

\section{Some quantitative And QUalitative Analysis}

Consider an Hamiltonian system

$$
\left\{\begin{array}{l}
\frac{\mathrm{d} q}{\mathrm{~d} t}=\frac{\partial H(q, p, t)}{\partial p}, \\
\frac{\mathrm{d} p}{\mathrm{~d} t}=-\frac{\partial H(q, p, t)}{\partial q} .
\end{array}\right.
$$

For the test, the Hamiltonian $H$ is chosen such that the solution is periodic. An approximate solution is computed within a period. The Euler explicit, a second and a forth Runge-Kutta, and the Borel-Padé-Laplace schemes are used. TABLE 3 shows the number of iterations of each method for some fixed precision. For example, to obtain a $10^{-6}$-precise solution, the resummation method requires 9 iterations. The other methods need far more iterations. Data on TABle 3 are plotted in Figure 2. The latter shows that the curve corresponding to the resummation-based scheme has the slowest slope when the precision is increased.

\begin{tabular}{|c|c|c|c|c|}
\hline Precision & Explicit & RK2 & RK & $\mathrm{BPL}$ \\
\hline $10^{-2}$ & 1904 & 65 & 10 & 3 \\
\hline $10^{-3}$ & 19042 & 199 & 17 & 4 \\
\hline $10^{-4}$ & 190400 & 629 & 30 & 5 \\
\hline $10^{-6}$ & $1.910^{7}$ & 6284 & 96 & 9 \\
\hline
\end{tabular}

TABLE 3. Required iteration numbers for $10^{-2}, 10^{-3}, 10^{-4}$ and $10^{-6}$-precise solutions

These tests shows that the resummation-base time-integration scheme is potentially very fast compared to the classical explicit numerical integrators. However, since the speed is not the only criterion for a good scheme, some geometric qualities of the scheme are analyzed. 


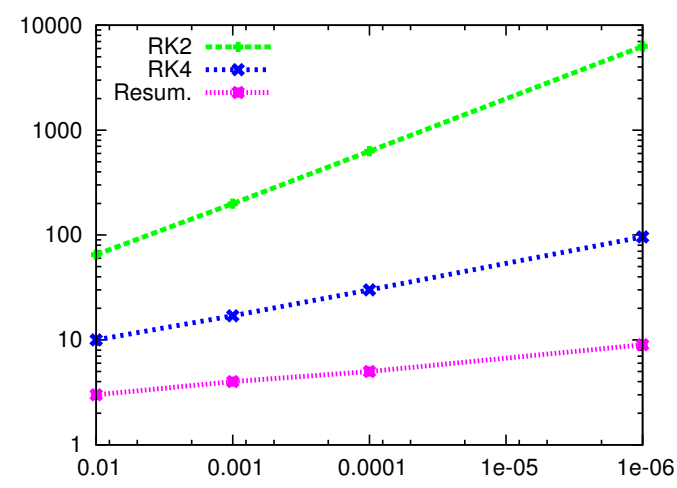

FiguRE 2. Evolution of the iteration numbers with increasing precision

Consider again the Hamiltonian system (9). With $H=\frac{1}{2}\left(p^{2}+q^{2}\right)$, the trajectory of each point is a closed circle. A non-optimized scheme may not be able to reproduce this property. For example, as seen in FiguRE 3 , left, the Euler explicit scheme introduces an error such that, at the end of each period, there is a shift and the trajectory looks more like a growing spiral rather than a circle. With the Borel-Padé-Laplace method, the non-closure of the trajectory is still almost invisible after 10 periods. The shape of the circle is well reproduced. For the figure, the initial point is $(q, p)=(1,0)$.
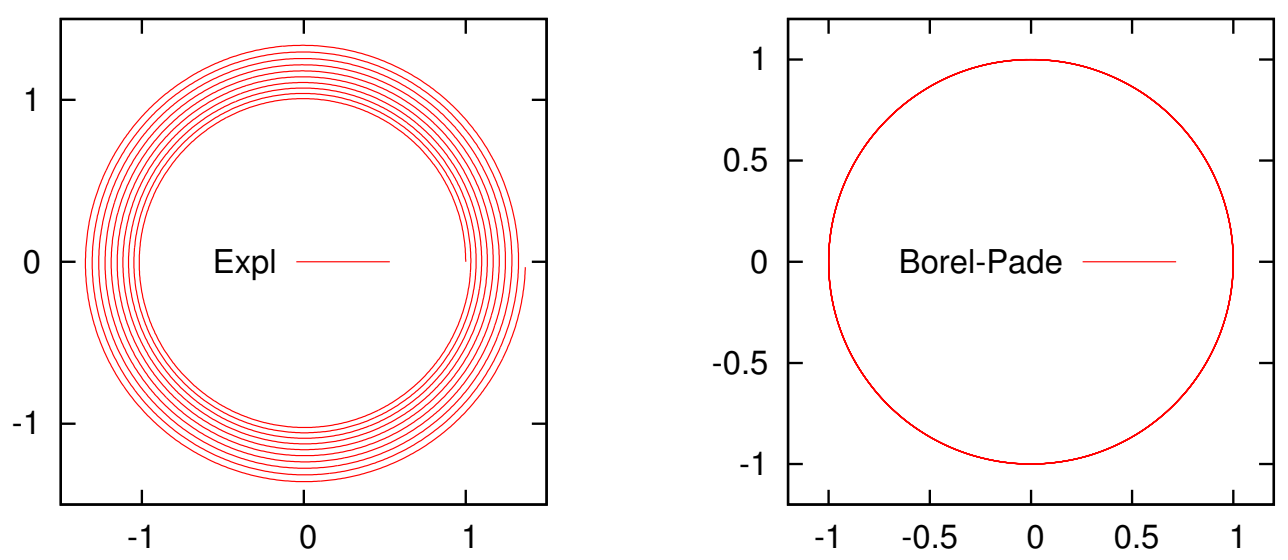

FiguRE 3. 10-period trajectory. Left, with the Euler explicit method. Right, with the resummation-based scheme.

Another important property of an Hamiltonian system is that any volume in the phase space is conserved along the time (Liouville's theorem). We check how good the resummation-based scheme respects this property.

We take as initial volume a 20-vertex regular polygonal, drawn in red in Figure 4. The evolution of the polygonal within ten periods is plotted in black. As seen on the left of the figure, the volume grows bigger and bigger with the Euler explicit method. With the resummation-based scheme, the polygonal does not exactly stay unchanged, but its volume remains approximately constant. This test shows the symplectic property of the scheme.

Note that the scheme was used as-is and, contrarily to other schemes (such as the symplectic Runge-Kutta), no effort has been made to make the scheme symplectic. 

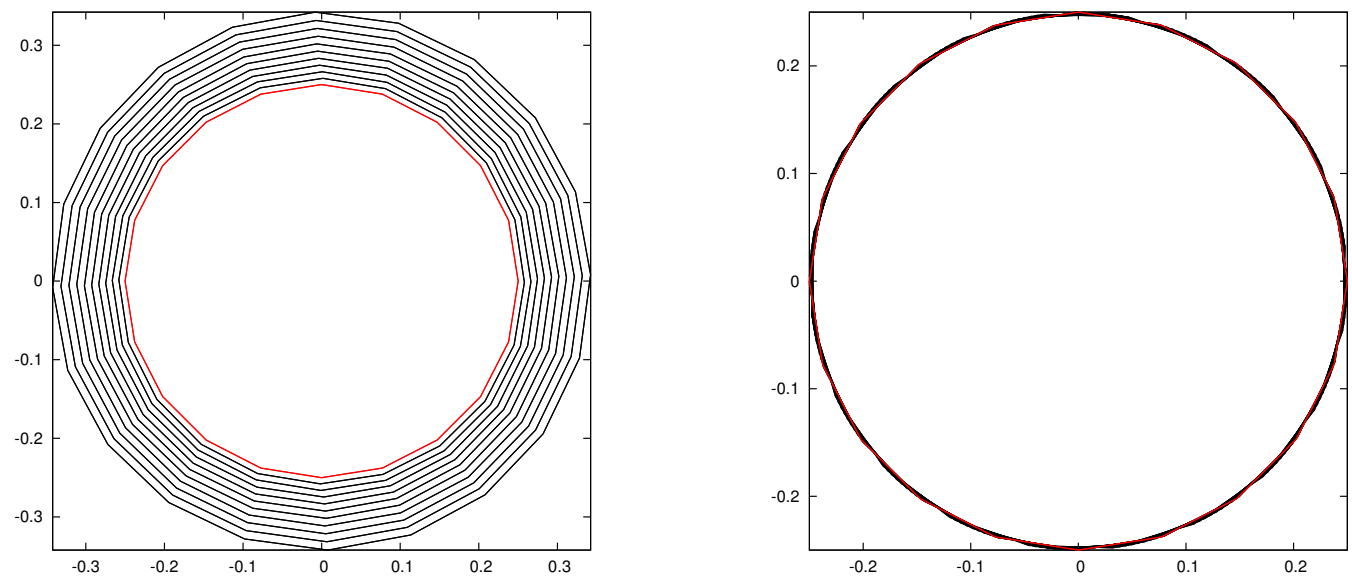

FIGURE 4. Evolution of a polygonal (in red) during 10 periods. Left, with the Euler explicit method. Right, with the Borel-Laplace-Padé scheme.

In the next test, we assume that system (9) is Liouville integrable. In this case, there exists a pair of operators $L$ and $M$, called a Lax pair, such that the Hamiltonian system is equivalent to

$$
\frac{\mathrm{d} L}{\mathrm{~d} t}=[L, M]
$$

In this equation, the brackets represent the commutator. It can be proven that $L$ is isospectral, that is, the eigenvalues of $L$ are time-independent.

For the test, $L, M$ and the initial condition are chosen such that the solution is

$$
L(t)=\left(\begin{array}{cc}
\tan \left(t+\frac{\pi}{4}\right) & 1 \\
-\cos ^{-2}\left(t+\frac{\pi}{4}\right) & -\tan \left(t+\frac{\pi}{4}\right)
\end{array}\right)
$$

The eigenvalues are $\lambda_{1}=i$ and $\lambda_{2}=-i$. Figure 5 represents the numerical evolution of the module of $\lambda_{1}$ with various schemes. It shows that, when $t$ gets close to the irregularity $\pi / 4$, the Euler explicit and the RungeKutta curves move away from the exact value $\left|\lambda_{1}\right|=1$. On the contrary, the resummation scheme crosses the irregularity without altering the module of $\lambda_{1}$.

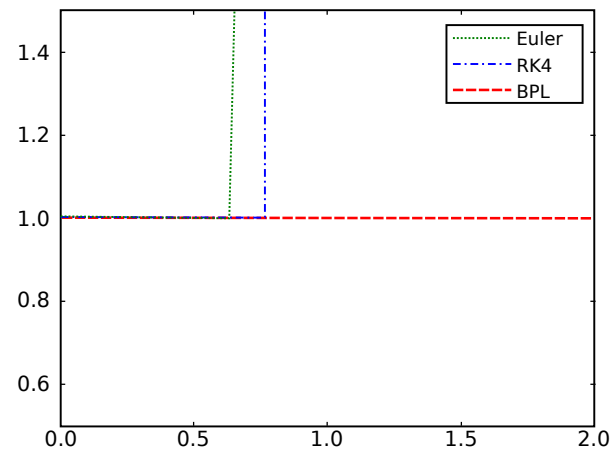

Figure 5. Module of eigenvalue $\lambda_{1}$ 
More generally, when $M$ is time independent, it is straightforward to prove the isospectrality of the scheme. Indeed, the formal series solution of $(10)$ is

$$
\hat{L}=\sum_{n \geq 0} L_{n} t^{n} \quad \text { with } \quad L_{n}=\frac{1}{n !} \sum_{k=0}^{n}(-1)^{k}\left(\begin{array}{l}
n \\
k
\end{array}\right) M^{n-k} L_{0} M^{k} .
$$

Hence,

$$
\operatorname{tr}\left(L_{n}\right)=\operatorname{tr}\left(M^{n} L_{0}\right) \sum_{k=0}^{n}(-1)^{k}\left(\begin{array}{l}
n \\
k
\end{array}\right)=0 \quad \forall n \geq 1 .
$$

Consequently, $\operatorname{tr}[\hat{L}(t)]=\operatorname{tr} L_{0}$ is time-independent. Similarly, it can be shown that $\operatorname{tr}\left[\hat{L}^{m}\right]=\operatorname{tr}\left[L_{0}^{m}\right]$ for any $m \in \mathbb{N}$. Since the eigenvalues are linear combinations of $\operatorname{tr}\left[\hat{L}^{m}\right]$, the approximate solution obtained with the asymptotic method is isospectral.

In the last section, some results on fluid mechanics problems are presented.

\section{Application to Fluid mechanics problems}

In [24], some tests on realistic fluid mechanics problem was presented. The Navier-Stokes equation was then reduced into a system of ordinary differential equations, thanks to the Proper Orthogonal Decomposition method [4,25]. And it was shown that the Borel-Padé-Laplace algorithm allows much bigger time steps than the Euler explicit scheme. In the present paper, we deal with the non reduced problem.

Consider the incompressible Navier-Stokes equations

$$
\left\{\begin{array}{l}
\frac{\partial u}{\partial t}+\operatorname{div}(u \otimes u)+\nabla p-\nu \Delta u=0 \\
\operatorname{div} u=0
\end{array}\right.
$$

along with an initial condition

$$
u(t=0)=u_{0}
$$

and boundary conditions.

We seek for a formal solution

$$
u(t)=\sum_{n \geq 0} u_{n} t^{n}, \quad p(t)=\sum_{n \geq 0} p_{n} t^{n} .
$$

As proven by Costin [12], the velocity and pressure series (16) are Gevrey series. When inserted in (14), series developments (16) lead to the recurrence relation:

$$
\left\{\begin{array}{l}
(n+1) u_{n+1}+\nabla p_{n}=-\sum_{k \geq 0}^{n} \operatorname{div}\left(u_{k} \otimes u_{n-k}\right)+\nu \Delta u_{n} \\
\operatorname{div} u_{n+1}=0
\end{array}\right.
$$

A projection-correction method is used for the computation of $u_{n+1}$ and $p_{n}$. The following pseudo-code shows how this is implemented. 


$$
\begin{aligned}
& \text { Solve } u_{*}=\frac{1}{n+1}\left[-\sum_{k \geq 0}^{n} \operatorname{div}\left(u_{k} \otimes u_{n-k}\right)+\nu \Delta u_{n}\right] \\
& \text { Solve } \Delta p_{n}=(n+1) \operatorname{div} u_{*} \\
& \text { Update } u_{n+1}=u_{*}-\frac{1}{n+1} \nabla p_{n}
\end{aligned}
$$

$u_{*}$ is an auxiliary variable. The terms $u_{n}$ are computed up to the desired order $N$, and $p_{n}$ up to $N-1$. And then, the resummation procedure is applied.

We choose a sized channel flow, periodic in stream- and spanwise directions (see FIGURE 6). A finite difference method is used for the space discretization.

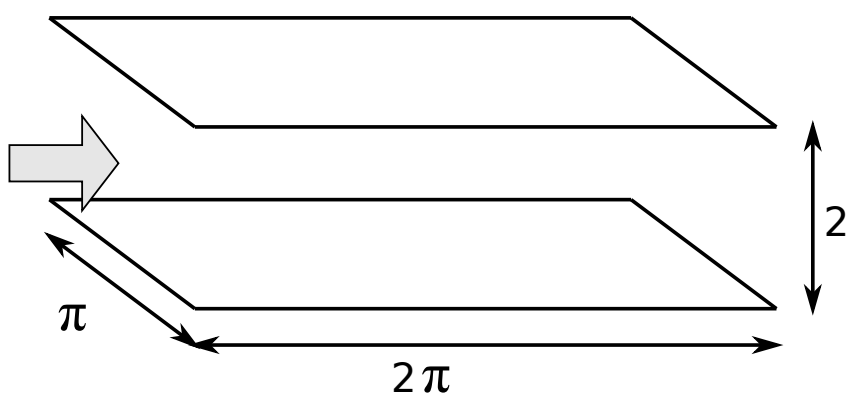

Figure 6. Channel flow configuration

In a first test, a source term is added such that the solution is periodic in time. More precisely,

$$
u=\cos (\pi t) \bar{u}
$$

where $\bar{u}$ has a Poiseuille profile. The approximate solution given by the series resummation approach is presented in Figure 7 where $N=8$. The solution obtained with an Adams-Bashforth scheme is also plotted for comparison.

In a second test, the source term is such that the solution is quasi-periodic in time:

$$
u=\left[\cos (10 \pi t)+\cos \left(10 \pi^{2} t\right)-1\right] \bar{u} .
$$

Figure 8 shows the approximate solutions. In these two test cases the series-based approximate solution is in a very good agreement with the exact one. Due to its high order, it is more precise than the Adams-Bashforth solution, especially near points where the curvature is high.

\section{Conclusion}

We showed that a time-integration scheme based on a series development and the Borel-resummation has interesting "spectral" properties. Moreover, tests on model differential equations shows that such an algorithm requires generally less time iterations than other classical explicit methods (see also [24]). However, for the non reduced Navier-Stokes equations, the number of iterations was not reduced. This is due to numerical difficulties to handling big numbers in the terms of the series. Optimization has to be carried out to overcome this problem. Other points can also be optimized. For example, the strength of the Borel-Padé-Laplace algorithm relies on 

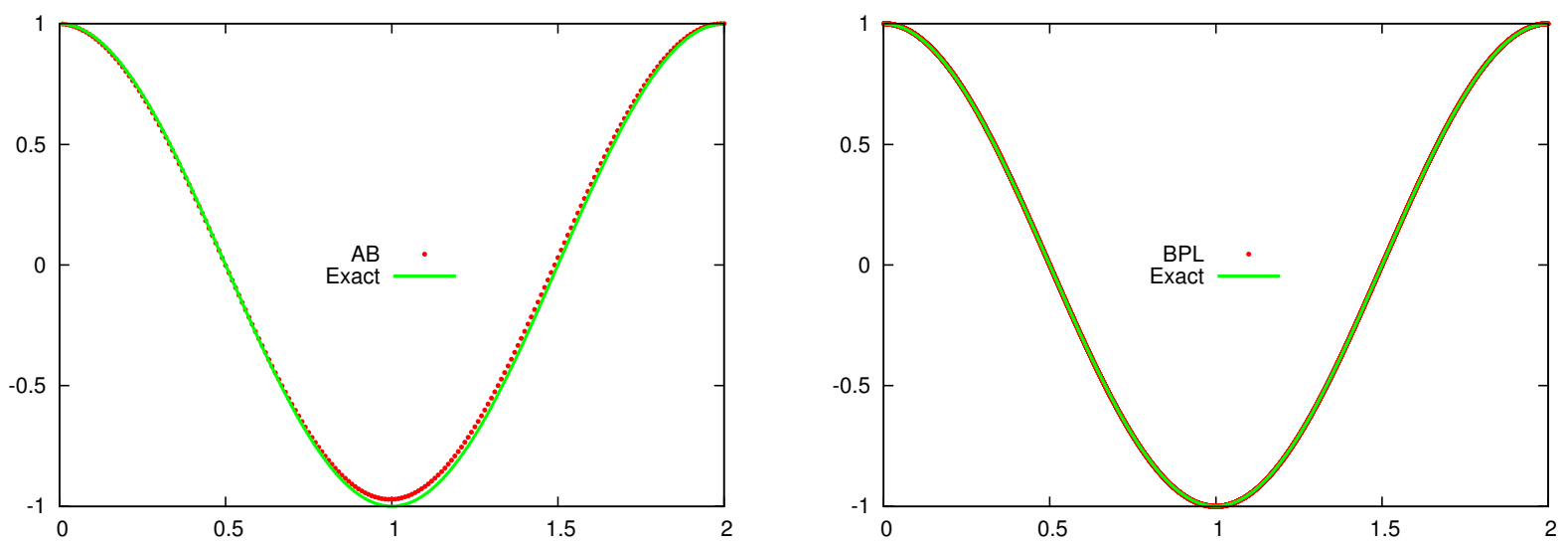

Figure 7. Time evolution of the approximate solutions in periodic case. Left: AdamsBashforth, right: Borel-Padé-Laplace
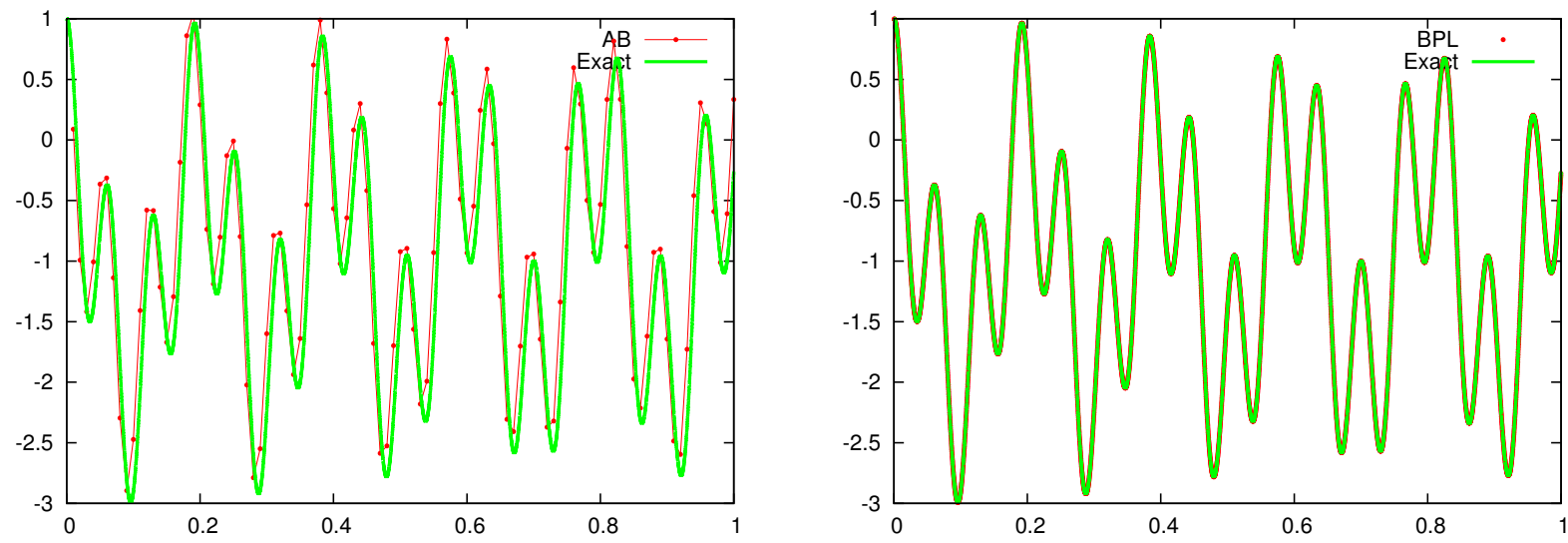

Figure 8. Time evolution of the approximate solutions in quasi-periodic case. Left: AdamsBashforth, right: Borel-Padé-Laplace

the efficiency of the Padé approximation in prolonging the Borel transformed series. This Padé approximation is however also an obstacle in the speed of the method. Indeed, in some cases, it has to be combined to other techniques (such as singular value decomposition method $[1,18]$ ) to avoid the apparition of a singular matrix. As an alternative, factorial series may be used $[15,27,28]$.

\section{REFERENCES}

[1] V. Adukov and O. Ibryaeva. A new algorithm for computing padé approximants. ArXiv, 1112.5694v, 2011.

[2] C. Allery, J-M. Cadou, A. Hamdouni, and D. Razafindralandy. Application of the asymptotic numerical method to the Coanda effect study. European Journal of Computational Mechanics, 13(1-2):57-77, 2004.

[3] M. Assidi, H. Zahrouni, N. Damil, and M. Potier-Ferry. Regularization and perturbation technique to solve plasticity problems. International Journal of Material Forming, 2(1):1-14, 2009.

[4] N. Aubry, P. Holmes, J. Lumley, and E. Stone. The dynamics of coherent structures in the wall region of a turbulent boundary layer. Journal of Fluid Mechanics, 192:115-173, 1988.

[5] C. Bender and S. Orszag. Advanced mathematical methods for scientists and engineers : Asymptotic methods and perturbation theory. McGraw-Hill, 1978.

[6] E. Borel. Mémoire sur les séries divergentes. Annales scientifiques de l'E.N.S. 3ème série, 16:9-131, 1899. 
[7] E. Borel. Leçons sur les séries divergentes. J. Gabay edition, 1928.

[8] C. Brezinski. Rationnal approximation to formal power serie. Journal of Approximation Theory, (25):295-317, 1979.

[9] C. Brezinski and J. Van Iseghem. Padé approximations. In P. G. Ciarlet and J. L. Lions, editors, Handbook of Numerical Analysis, volume 3, pages $47-222$. Elsevier, 1994.

[10] J.M. Cadou, M. Potier-Ferry, and B. Cochelin. A numerical method for the computation of bifurcation points in fluid mechanics. European Journal of Mechanics - B/Fluids, 25:234-254, 2006.

[11] O. Costin. Asymptotics and Borel Summability. Monographs and Surveys in Pure and Applied Mathematics. CRC Press, 2008.

[12] O. Costin and S. Tanveer. Borel summability of Navier-Stokes equation in $\mathbb{R}^{3}$ and small time existence. arXiv:math/0612063, 2006.

[13] N. Damil and M. Potier-Ferry. A new method to compute perturbed bifurcations : application to the buckling of imperfect elastic structures. International Journal of Engineering Science, 28(9):943-957, 1990.

[14] L. Daridon, B. Wattrisse, A. Chrysochoos, and M. Potier-Ferry. Solving fracture problems using an asymptotic numerical method. Computers and Structures, 89(5):476-484, 2011.

[15] E. Delabaere and J-M. Rasoamanana. Sommation effective d'une somme de Borel par séries de factorielles. Annales de l'institut Fourier, 57(2):421-456, 2007.

[16] F. J. Dyson. Divergence of perturbation theory in quantum electrodynamics. Physical Review, 85:631-632, 1952.

[17] M. Gevrey. Sur la nature analytique des solutions des équations aux dérivées partielles. Premier mémoire. Annales scientifiques de l'E.N.S. $3^{\text {ème }}$ série, 35:129-190, 1918.

[18] P. Gonnet, S. Güttel, and L. Trefethen. Robust Padé approximation via SVD. SIAM Review, 51(1):101-117, 2013.

[19] G. Kontopoulos. Order and chaos in dynamical astronomy. Astronomy and astrophysics library. Springer, 2002.

[20] D.A. Lutz, M. Miyake, and R. Schäfke. On the Borel summability of divergent solutions of the heat equation. Nagoya Mathematical Journal, 154:1-29, 1999.

[21] J. Ramis. Les series $k$-sommables et leurs applications. In D. Iagolnitzer, editor, Complex Analysis, Microlocal Calculus and Relativistic Quantum Theory, volume 126 of Lecture Notes in Physics, pages 178-199. Springer Berlin / Heidelberg, 1980.

[22] J-P. Ramis. Séries divergentes et théories asymptotiques. In Journées X-UPS 1991, pages 7-67, 1991.

[23] J-P. Ramis. Poincaré et les développements asymptotiques (première partie). Gazettes des Mathématiciens, (133), 2012.

[24] D. Razafindralandy and A. Hamdouni. Time integration algorithm based on divergent series resummation, for ordinary and partial differential equations. Journal of Computational Physics, 236:56-73, 2013.

[25] L. Sirovich. Turbulence and the dynamics of coherent structures, Part I: Coherent strucures, Part II : Symmetries and transformations, Part III : Dynamics and scaling. Quarterly of Applied Machanics, 45(3):561-590, 1987.

[26] I Suslov. Divergent perturbation series. Journal of Experimental and Theoretical Physics, $100,2005$. ArXiv:hep-ph/0510142.

[27] J. Thomann. Procédés formels et numériques de sommation de séries solutions d'équations différentielles. In Centre de mathématiques, editor, Journées X-UPS 1991, Séries divergentes et procédés de resommation, pages $101-114$, 1991.

[28] E. Weniger. Summation of divergent power series by means of factorial series. arXiv:1005.0466 [math.NA], 2010. 